\title{
Musik-Filmische Teilhabekonstellationen als Partizipationsversprechen und situiertes Wissen in The Queen of Silence (2014) und And-Ek Ghes... (2016) ${ }^{1}$
}

\begin{abstract}
Der Beitrag geht der Frage nach, wie (illegitime) städtische Räume zu Schauplätzen einer Reflexion von Teilhabe werden können, wenn sich Prozesse des Filmemachens, Tanzens und Musik-Hörens miteinander verschränken. Es wird dafür argumentiert, Partizipation im Kontext dokumentarischer Bewegtbild-Produktionen als prozessual wie auch medial hergestellte Teilhabe zu konzeptualisieren. Die Analyse audiovisueller Teilhabekonstellationen, durch die sonische Körper mit konstituiert werden, konzentriert sich auf zwei Filme: In The Queen of Silence (Agnieszka Zwiefka) und And-Ek Ghes... (Philip Scheffner und Colorado Velcu) kommen Musikvideo-Ästhetik und Referenzen auf Bollywood eine entscheidende Rolle zu. Dabei stellt sich die Frage, wie diese Produktionen Repräsentationspolitiken hinsichtlich der Sinti und Roma in Polen und Deutschland durchdenken. Die Resonanzen zwischen Musik, Bewegtbild, tanzenden/hörenden Körpern und urbanen Räumen werden mit Blick auf die Sound Studies und Disability Studies kartiert und die Frage nach den Möglichkeiten einer situierten Wissensproduktion (Haraway) erörtert.
\end{abstract}

\section{Einleitung}

Die fünfte Folge der Dokumentar-Miniserie „Sound System“ (Frankreich 2016), ausgestrahlt vom Sender arte, trägt den Titel „Rastaman Migration“. Die Produktion zeichnet die Entstehung und den Weg von Soundsystemen nach und begibt sich dafür in Städte der Karibik, den USA, Südamerikas, Asiens und Europas. Einer der Orte, an dem sich die globale Story von Dancehall-Klängen verdichtet, ist London. Der dort seit den 1960er Jahren stattfindende Notting Hill Carnival kann als ein exemplarisches Beispiel für die Art begriffen werden, wie sich im postkolonialen Vereinigten Königreich soziale Zugehörigkeiten, Musikkulturen, Kostüme, Beschallungssysteme und städtische Räume sowie deren Kommerzialisierung oder Gentrifizierung prozessual herausbilden und performativ miteinander verweben (Klöß 2014, S. 451-461). Diesen konfliktreichen Aushandlungsprozess, der sich mit dem Karneval verbindet, bezeichnet Paul Gilroy mit Blick auf die 1970er und

1 Dieses Kapitel entstand im Rahmen des Projekts „Recht auf Mitsprache: Das Cochlea-Implantat und die Zumutungen des Hörens“, Teilprojekt 2 der Forschungsgruppe „Mediale Teilhabe. Partizipation zwischen Anspruch und Inanspruchnahme“, gefördert von der Deutschen Forschungsgemeinschaft (DFG), Projektnummer 272138722. 
1980er Jahre als „,kind of recolonisation. People had never seen the costumes before, had never seen dancing in the streets like that" (zit. n. Procter 2003, S. 85). Die Konfrontation, die Gilroy hier andeutet, verweist auf die Begegnung und die Spannungen sowie auch die gewaltsamen Kämpfe zwischen KarnevalsteilnehmerInnen und den Ordnungsbehörden, die die Veranstaltung beaufsichtigten. Im Hinblick auf jüngere Entwicklungen stellt die erwähnte Miniserie dies ebenso in den Vordergrund. Gilroy kritisiert im Interview, dass MigrantInnen aus der Karibik zwar einen englischen Pass hätten, aber ihnen keine „öffentlichen Räume“ in der Stadt zugestanden würden. Diese würden sie etwa mit dem Karneval und den Sound Systemen selbst erschaffen. Doch Ladbroke Grove, Teil von Notting Hill, hätte sich in den vergangenen Jahrzehnten zunehmend von einem armen Stadtteil in ein Viertel für gutsituierte Menschen der Oberschicht transformiert. Womit sich der Karneval nun vor einer Szenerie verortet, in der Wohnhäuser oft mit Sperrholzplatten gegen die mittlerweile kommerzialisierte Partykultur - auch mit seinem „family day“ - abgegrenzt werden.

Die Konfrontationen und Spannungen, die sich mit dem Notting Hill Carnival verbinden und auf die hier nur kursorisch hingewiesen werden kann, weisen nicht nur auf eine spezifische Situation von Enteignet-Sein oder Enteignet-Werden (Athanasiou/Butler 2014) hin. Darüber hinaus machen sie auf unterschiedliche Rhythmen und Klangstrukturen in einer Großstadt wie London aufmerksam. Wie Lefebvre herausgestellt hat, sind Orte und Zeiten in Städten durch spezifische Rhythmen geprägt: „Rhythm is always linked to such and such a place, to its place, be that the heart, the fluttering of the eyelids, the movement of a street or the tempo of a waltz. This does not prevent it from being a time, which is to say an aspect of a movement or of a becoming" (Lefebvre 2004, S. 89). Die Herausbildung solcher Rhythmen beruht auf Wiederholung, wie Deleuze und Guattari (1987, S. 426f.) in Tausend Plateaus beschrieben haben: „Jedes Milieu vibriert, das heißt, es ist ein Block aus Raum und Zeit, der durch die periodische Wiederholung der Komponente gebildet wird." Im erwähnten Karneval werden folglich gegenwärtig die Rhythmen des ,ruhigen" Oberschichtsviertels mit denen der Dancehall-Vibes und der klanglichen Dominanz der Sound Systeme gekreuzt. So werden nicht nur heterogene Elemente durch Rhythmus miteinander in Beziehung gesetzt (Turetzky 2002, S. 124). In dieser Anordnung ist zu beobachten, wie sich sonische Körper im Zusammenwirken von Soundsystemen, Klang, Rhythmus, und Menschen in Bewegung in spezifischen räumlich wie auch zeitlichen Verortungen formen (Henriques 2011). Das performative Potenzial dieses Geschehens kann dabei freilich nicht von asymmetrischen Machtbeziehungen getrennt gesehen werden, wie sie im Rahmen der Aufteilung des Sinnlichen (Rancière 2006) oder einer „Politik der Straße“, die prekäre Lebensbedingungen, wirtschaftliche Ungleichheit, Rassismus auf die Tagesordnung bringt (Butler/Athanasiou 2014, S. 49, 56), zum Vorschein kommen.

Diese Überlegungen sollen im Folgenden auf den Bereich des Dokumentarfilms (Hohenberger 2006) bezogen werden. Im Hinblick auf sonische Körper und die Analyse von Rhythmen kommen folglich Aspekte hinzu, die die Kadrierung körperlicher Performativität sowie die Produktion des (filmischen) Hörens betreffen, 
wobei letzteres Chion (1994, S. 136) zufolge als transsensorischer Prozess begriffen wird. Die Frage lautet, in welchen filmischen Formen inner- und außerstädtische sowie auch als illegitim angesehene Räume zu Ausgangspunkten und Schauplätzen für eine Reflexion von Teilhabe werden, in denen Prozesse des Filmemachens, Tanzens und Musik-Hörens - sowie unterschiedliche Rhythmen - thematisiert werden. Es soll eine kritisch-reflektierte Sichtweise auf Filmproduktionen entwickelt werden, die versuchen ihre Wirklichkeitsversionen anhand ,partizipativer“ Ansätze (Harper u.a. 2016; Rogers 2016; Sudbury 2016; Milne/Mitchell/De Lange 2012; Ruby 1992) umzusetzen. Ausgangspunkt ist dabei die Annahme, dass Partizipation im Kontext dokumentarischer Bewegtbild-Produktionen als prozessual wie auch medial hergestellte Teilhabe begriffen werden muss. Im Aufeinandertreffen von Filmtechnologien, Gefilmten und Filmenden, von Kostümen, Klängen und Rhythmen sowie urbanen Orten werden Teilhabebeziehungen gestiftet und Machtrelationen bzw. deren Reflexion beobachtbar (vgl. Bippus/Ochsner/Otto 2016). Um dies aufzuzeigen, fokussiert die Analyse zwei Filme, an denen sich die Problematik audiovisueller Teilhabekonstellationen und sonischer Körper eindrücklich demonstrieren lässt: The Queen of Silence von Agnieszka Zwiefka und And-Ek Ghes... von Philip Scheffner und Colorado Velcu. In beiden Produktionen spielen Musikvideo-Ästhetik (Bergermann 2003; Keazor/Wübbena 2017) und Referenzen auf Bollywood (Gehlawat 2015) eine entscheidende Rolle. Zu berücksichtigen ist allerdings, dass diese Filme sich auf unterschiedliche Weise mit den Repräsentationspolitiken hinsichtlich der Sinti und Roma in Polen und Deutschland auseinandersetzen (vgl. Bogdal 2011; Brunner 2017). Die verschiedenen Ebenen von Musik, Bewegtbild und tanzenden/hörenden Körpern werden im Folgenden anhand der genannten Filme einer medienkulturwissenschaftlichen Analyse zugeführt, die Brücken zu den Sound Studies und Disability Studies schlägt (vgl. Friedner/Helmreich 2012).

\section{The Queen of Silence}

\section{Resonanzen zwischen einer Roma-Siedlung und der Stadt Wroclaw}

Der Vorspann des Films The Queen of Silence zeigt die Protagonistin Denisa Gabor im Wechsel in ihrem Zuhause und in einer Hörtest-Kabine mit ihren Eltern. Beide Orte werden über den Soundtrack durch langsame Sinustöne verbunden, die auf die Hörbehinderung der Protagonistin verweisen. So wird einerseits auf die Schwerhörigkeit inklusive ihrer medizinischen Diagnose durch die Audiologin aufmerksam gemacht, wobei eine klinisch-neutrale Atmosphäre vorherrscht und das Mädchen den Test sitzend erledigen muss. Andererseits verweisen die Einstellungen in der provisorischen Unterkunft der illegal(isiert)en Roma-Siedlung darauf, dass rhythmische Erfahrungen und Bewegungen nicht von einem (physiologisch) ,normalen' Hören abhängig sein müssen. In Nahaufnahmen werden wiederholt die mit unzähligen glänzenden Ringen versehenen Arme der Protagonistin gezeigt, die sich beständig gegenseitig berühren und durch die Luft gleiten. Dabei 
entstehen perkussionsartige und rhythmische Metallklänge, die die in nachmittägliches Sonnenlicht getauchte Szenerie füllen. Daneben kommen auch die unbekleideten Füße des Mädchens ins Bild. Sie stehen in direktem Kontakt mit dem Boden, berühren diesen und geben rhythmische Impulse. Es sind, wenn man so möchte, taktile Bilder, die Berührungspotenziale und -wirkungen dynamisch vermitteln (Harrasser 2017). Zugleich zeichnen sie sich durch auditive Qualitäten aus. Die verschiedenen Sinne werden somit auf spezifische Weise je nach Setting unentwirrbar miteinander verwoben. Dadurch zeichnet sich die Erfahrung eines tanzenden und sonischen (Henriques 2011), eines sinnlich empfindsamen Körpers ab, der scheinbar außerhalb des ärztlichen Zugriffs und jenseits defizitärer Zuschreibungen im Sinne eines medizinischen Modells von Behinderung (Waldschmidt 2015) performiert wird. Zugleich wird im Zusammenhang der - illegitimen - Siedlung auch das den Sinti und Roma zugeschriebene ,romantische Klischee der angeborenen Musikalität“" (Brunner 2017, S. 277) in gewisser Weise aufgerufen und verschoben.

Schnitt - Beginn des Films: Denisa Gabor erscheint vor der Kamera, sie gestikuliert und spricht. Ihre lautsprachlichen Äußerungen sind nicht verständlich. Auf einem Sperrmüllcontainer sitzend zeigt sie der Kamera eine Barbiepuppe: Im Hintergrund ist die Roma-Siedlung zu sehen. Sie säubert die gefundene Barbie und spielt mit ihr, was der Film in einer langen Einstellung zeigt. Darauf sehen wir das Mädchen beim Spielen mit anderen Kindern in der Siedlung. In abfälliger Weise wird sie von einem Jungen als „Taube“ bezeichnet. ${ }^{2}$ Diese Szenen setzen eine kindliche Perspektive auf das prekäre Leben in der Roma-Siedlung außerhalb von Wrocław ins Bild. Auch ihre Situation als Mädchen mit einer Hörbehinderung, ihre Art für sich zu sein und die Schwierigkeiten im Miteinander mit Gleichaltrigen werden thematisiert. Zugleich stellen diese Szenen eine Herausforderung für ein auf Sprachverständnis ausgerichtetes Hören dar. Denn die Äußerungen der Protagonistin bleiben unverständlich, auch wenn deren möglicher Sinn - die Entdeckung der Puppe und das Spiel mit ihr - offensichtlich erscheinen mag. ${ }^{3}$ Somit sind wir hier mit einer filmischen Produktion von „crippled speech“ (Marshall 2014) konfrontiert, die durch verbale Artikulation und das Hören zugleich entsteht. ${ }^{4}$

Das Mädchen Denisa, ihre verbalen Äußerungen und ihr Handeln mit der Kamera werden zum Ausgangspunkt einer wechselseitigen Beziehungsarbeit, die den

2 Die Bezeichnung ,taub“ generiert in der betreffenden Szene eine Form von Ausschluss, ist aber nicht generell in dieser Weise festgeschrieben. Zu Differenzierungen und Verwendung des Begriffs durch Gehörlose vgl. Uhlig 2012, S. 339f.

3 Eine Hörbehinderung ist in der Regel mit einem verlangsamten Sprachlernprozess verbunden. Denisas Schwerhörigkeit wurde erst spät diagnostiziert, weshalb sie kein Romanes spricht.

4 Marshall (2014) führt dazu aus: ,,[...] crippled speech is understood through a particular mode of listening and hearing [...] Crippled speech is thus brought into being by the disabling environment created by normative hearing. To cripple speech is a technology of power that, when applied, marks vocal difference, whether that difference is perceived as racialized timbre, queered inflection, deaf accent, or dystonic slurring. The term 'crippled speech' describes both the norm of a discriminatory mode of listening and hearing, and the markedness of non-normative utterances." 
gesamten Film The Queen of Silence durchzieht. ${ }^{5}$ Die Filmemacherin Agnzieszka Zwiefka beschreibt, dass sich Denisa ihnen förmlich als Protagonistin aufdrängte, als sie eines Tages in der Siedlung erschien. „Either it is a film about her or no film at all" (Zwiefka in Katie chats). Zu diesem Zeitpunkt war Zwiefka schon ein knappes Jahr vor Ort. Doch mit Denisa änderte sich alles. Genau auf diese Konstellation scheinen viele der Szene zu verweisen: Das Mädchen richtet sich an die Kamera, nimmt Kontakt auf und will, dass sich ihre und die Welt des Films berühren. Für Denisa mit einer Hörbehinderung und ohne Kenntnisse der Gebärdensprache sei die Kamera ein wichtiges Element, um mit der „Welt“ zu kommunizieren (Katie chats). ${ }^{6}$

The Queen of Silence sei ein Film über Träume, so Zwiefka. Es sei ein Film über „eine Flucht vor der Realität“" (Katie chats), der in der Konsequenz in seinem Aufbau zweigeteilt ist: Einerseits bedient er sich der Elemente des observational cinema und sucht ein Bild vom Alltag in und um die Roma Siedlung zu entwerfen. Kochen, putzen in der Siedlung, um Geld bitten in den Straßen der Innenstadt oder das Durchsuchen von Altkleidercontainern sind Aktivitäten der Gefilmten, die der Film hervorhebt und dabei einer stereotypisierenden Darstellung nicht immer ausweichen kann. ${ }^{7}$ Die Flucht aus dieser (filmischen) Realität wird andererseits durch mehrere choreografierte Musikvideos artikuliert. Diese stehen stilistisch in Kontrast zum eher dokumentarischen Ansatz (Festival dei Popoli, 00:03:45), wie gleich näher gezeigt wird.

Ein verbindendes Element der beiden Sphären - der Armut in der RomaSiedlung und einem idealisierten Leben als Bürgerin der Stadt Wrocław - bildet der Erzählstrang, in dem es um die Behandlung von Denisas Hörbehinderung geht.

5 Zwiefka beschreibt, dass sie über einen Zeitraum von rund drei Jahren mit Denisa Gabor zusammenarbeitete. Sie konnten sich nicht über Lautsprache verständigen. Die Regisseurin schildert aber, wie sie einen Weg fanden zu kommunizieren. Denn das Mädchen ,invented her own sign language“" und diese reduzierte Sprache, die sie zur Verständigung mit ihrer Mutter nutzt, half auch Zwiefka mit dem jungen Mädchen zu kommunizieren (Festival dei Popoli, 00:04:12).

6 Zwiefka zeigte den Film auch den Roma in der Siedlung. Diese waren laut der Filmemacherin vom Ergebnis begeistert, obwohl sie nicht immer im besten Licht gezeigt werden. Die Beziehung von Zwiefka hatte darauf möglicherweise auch einen Einfluss, hatte sie doch über Jahre mit den Roma dieser Siedlung gearbeitet und wurde nicht mehr als Bedrohung angesehen, sondern vielmehr als jemand, der versuchte ihre Lebensweise zu begreifen und darüber einen Film zu machen. Diese Beziehung aufzubauen war jedoch nicht einfach. So war in den Augen der Filmemacherin das erste Jahr der Filmarbeit sehr schwierig. Die BewohnerInnen seien gute SchauspielerInnen und hätten sie nicht wirklich an ihr Leben herangelassen. Erst nach einer Zeit entstand ein gewisses Vertrauensverhältnis, das es der Filmemacherin erlaubte, genau diesen Film zu realisieren (Festival dei Popoli, 00:06:40).

7 Zur Situation der Roma in Europa, aber speziell auch in Polen vgl. das Dossier „Sinti und Roma“ der Bundeszentrale für politische Bildung. Siehe auch darin Blecking (2014). Brunner notiert: „Die Wahrnehmung der Roma - in der abwertenden Fremdbezeichnung als ,Zigeuner' benannt ist stark von Stereotypen und Vorurteilen geprägt. Dies betrifft nicht nur ihren vermeintlichen Lebensstil des Vagabundierens, ein Bild, das sich hartnäckig hält, obwohl Roma durchwegs sesshaft sind, oder deren angeblichen Hang zu Diebstahl oder Trickbetrug. Ebenso beständig ist das romantische Klischee der angeborenen Musikalität, also die Vorstellung, dass Roma die ,Musik im Blut‘ hätten“" (2017, S. 276f.). 
So soll durch die Technologie des Hörgeräts ein Kontakt ermöglicht werden zwischen der Stadt mit ihrem Rhythmus aus Infrastrukturen, sozialen Einrichtungen, Ausbildungsmöglichkeiten und medizinischen Versorgung einerseits und der Roma-Siedlung mit ihrem Rhythmus aus provisorischen Bauten, fehlender langfristiger Sicherheit sowie prekären Lebensverhältnissen andererseits. In dieser recht dichotom wirkenden Gegenüberstellung soll dem Hörgerät, dem Hören lernen und den Sprachübungen eine vermittelnde Funktion zukommen. Die mit Hörgerät ausgestattete Denisa lernt zwar teils ein verstehendes Hören, was der Film effektvoll in einer Szene inszeniert. Doch es bleibt bei einem Versuch, der sich letztlich nicht als erfolgreich erweist. ${ }^{8}$ Es bleibt bei einem flüchtigen Kontakt der beiden vibrierenden Milieus. Die ermöglichte Resonanz verhallt alsbald bzw. wird in andere Ebenen des Films transponiert. ${ }^{9}$

Wie schon angedeutet, zeichnet sich The Queen of Silence nicht nur durch seinen Fokus auf Denisa Gabor aus. Besonders hervorzuheben sind auch die Tanzszenen und Bollywood-Referenzen. Diese Schwerpunktsetzung geht auf die Initiative der Protagonistin zurück. So betont Zwiefka die Zusammenarbeit mit der Protagonistin als wichtiges Element im Entstehungsprozess des Films: „The idea, the concept of a film should come from the protagonist, it should not be imposed by the director him or herself" (Festival dei Popoli, 00:01:50). Im Folgenden soll diese Form der Zusammenarbeit und ebenso die Ästhetik der Musikvideos näher untersucht werden. Dabei verfolge ich die Frage, inwiefern durch die Musik, die Choreographie sowie auch die filmische Ins-Bild-Setzung eine Teilhabekonstellation entworfen wird, die die Aneignung innerstädtischer durch die - ausgegrenzten Roma in Aussicht stellt, zugleich aber auch eine Reihe von Widersprüchen mit sich bringt.

\section{Ein Märchen über Partizipation}

Eine längere Sequenz in der ersten Hälfte des Films zeigt Denisa Gabor und ein weiteres Mädchen dabei, wie sie einen Altkleidercontainer durchsuchen und die

8 Es kann hier nur darüber spekuliert werden, warum der Einsatz des Hörgeräts sich als nicht erfolgreich herausstellt. Einerseits sind mit einer solchen empfindlichen Technologie bestimmte Handhabungsweisen verbunden, die sich im Kontext der Siedlung möglicherweise als nicht realisierbar erweisen. Auch das tägliche Üben von Sprachverstehen ist bei Kindern, die nicht oder unregelmäßig die Schule besuchen, bildet ein potenzielles Hindernis. Darüber hinaus ist aber auch zu berücksichtigen, dass die Normalisierung von Denisas Hören ihre Integration in die ökonomischen Praktiken der Roma zur Folge hätte. Doch sie weigert sich, im Stadtzentrum um Geld zu betteln (so stellt es zumindest der Film dar). Auch der gescheiterte Versuch das Hören zu erlernen, ist unter Umständen darauf zurückzuführen. Doch das ist spekulativ.

9 Denisa wurde von Eltern zurück nach Rumänien geschickt, Filmemacherin setzt sich für sie ein, organisiert u.a. Internatsplatz für sie in einer Schule für Gehörlose. Es bestand zudem die potenzielle Möglichkeit für Denisa an einer Musical-Produktion teilzunehmen. Zwiefka möchte finanzielle Hilfe organisieren, denn Denisa ging nie zur Schule und sie möchte sich für sie einsetzen, damit sie ihren Weg gehen kann (Katie chats). 
gefundenen Dinge in die Siedlung bringen. Dort entdeckt die Protagonistin die DVD eines Bollywood-Films, die sie zusammen mit ihrem Bruder und der Mutter in deren Hütte schauen. Die Kamera konzentriert sich dabei auf Denisa, wie sie den Film sieht und beginnt die Choreographien nachzuahmen. Hier wird deutlich gemacht, wie eng das Leben in der Siedlung und die „escape from harsh reality“ (Zwiefka in Katie chats) zusammenhängen. Die Bollywood-Produktionen werden für Denisa zu einer Art Refugium, die eine Abwechslung zum Alltag bieten. Die Faszination der Kinder für Bollywood wird noch an anderer Stelle deutlich: Von der Kamera beobachtet sehen mehrere Kinder die DVD im Fernsehen. Die Protagonistin und ein anderes Mädchen tanzen zu den Choreographien. Wichtig ist hier der Soundtrack: Denn es wird vom on-screen Ton des flach tönenden Fernsehers und der sich unterhaltenden Kinder plötzlich auf den direkten Sound der DVD umgeschaltet. Der somit entstehende Effekt macht die Szene vom dokumentarischen Regime unterscheidbar und zeigt mithin an, dass sich die Kinder temporär ein Stück weit vom Leben der Siedlung entfernen und so den idealisierten Darstellungen des gesehenen Films anhängen. Die Blicke der Kinder richten sich auf den Fernseher im Sinne einer „Klangmauer“ (Deleuze/Guattari 1987, S. 424), die ein imaginäres Territorium absteckt. Die Körper der zwei Mädchen (die der Jungen hier noch nicht) werden von der Musik in Bewegung versetzt. Auf diese Weise emergiert ein temporärer filmisch-televisueller Klang-Raum, in dem das Auditorische, Rhythmus und Körper in Bewegung miteinander gekoppelt werden (Henriques 2011).

Die nachfolgenden in den Film eingebrachten Musikvideos entwerfen dann eine Bewegung, in der eine Teilhabe der Roma-Kinder an den innerstädtischen Lebenswelten in Aussicht gestellt wird. Dies wird in mehreren Schritten vorgeführt: Das erste Tanz-Video hat die prekäre Roma-Siedlung als Schauplatz (00:22:3000:23:47): Denisas Mutter streitet mit der Nachbarin, währenddessen kommt die Protagonistin ins Bild. Der onscreen Ton wird ausgeblendet, die streitenden Frauen stumm gestellt. Schnell geschnittene Szenen vom Wäschewaschen in der Siedlung, die mit Slow Motion-Einstellungen durchsetzt sind. In der Folge tanzen Jungen und Mädchen mit bunten Tüchern als Accessoires zur populären Roma-Musik von Dani Printul Banatuluis Titel „Tranquila“, der musikalisch zwar an Klänge der Balkanbeats à la Shantel und deren Mix mit elektronischer Tanzmusik anknüpft (Brunner 2017, S. 280), diese nun aber ohne Blasmusik-Kapelle und in einer Art Mainstream-Variante mit Gangsta-Rap-Anleihen präsentiert. Die knallige Farbigkeit der Tücher in Kombination mit den choreografierten Bewegungsabläufen schreibt den Clip wiederum in die Ästhetik von Bollywood-Szenarien ein, die die Kinder im Film wiederholt auf dem TV-Bildschirm beobachten. Im zweiten Videoclip wird eine Begegnung mit der polnischen Staatsmacht inszeniert, nach dem die Polizei in der Siedlung nach zwei Personen gesucht hat, für die ein Gerichtsbescheid vorliegt. Die Kinder, dieses Mal mit Spielzeugpistolen ausgestattet, sehen sich darin tanzend einem Polizisten gegenüber. Die nachgespielte Konfrontation mit der Polizei wird mit einem Titel des bereits erwähnten Banatului - „Esti bomba“ - unterlegt. Nun überwiegen rhythmische Percussion-Klänge, die die Grenzen zwischen Roma und 
Bollywood-Musik verschwimmen lassen. Zudem wird ein Verfolgungsszenario performiert, das in gewisser Weise Assoziationen mit Slum Dog Millionaire (2008) von Danny Boyle weckt (Tzanelli 2016). Die rasante Videosequenz endet schließlich mit einem für die Hand-Kamera inszenierten Rollenspiel, bei dem Jungen aus der Siedlung Denisa gefangen nehmen und ihr mit der Abschiebung nach Rumänien drohen. So wird in einer Rückkehr in einen dokumentarisch-beobachtenden Modus die Frage nach dem unsicheren Aufenthaltsstatus der Sinti und Roma in Polen aufgeworfen (vgl. u.a. Koch 2010, S. 270-274; Bunescu 2014; Trauschein 2014; Fings 2016).

Während die oben besprochenen Musikvideos sich auf den Raum der RomaSiedlung beziehen, ist in der dritten Choreografie ein Ortswechsel festzustellen. Das Setting ist nunmehr die Vorstadt von Wrocław. Denisa Gabor thront als Königin mit goldener Papierkrone auf einem Sessel aus dem Sperrmüll. So wird sie von drei Jungen und ihrer Freundin auf einen Sportplatz in der Neubausiedlung getragen. Dort dirigiert sie zuerst eine ganze Schar von Kindern aus der Vorstadt und der Roma-Siedlung, die gemeinsam zur Musik von Banatului tanzen. Im zweiten Part des Clips gibt Denisa dann einer Gruppe von jugendlichen Tänzern Anweisungen auf dem Basketball-Platz. Die Kamera zeigt Denisa hier ebenfalls in einer erhöhten Position, von der aus sie das Geschehen bestimmt. Im dritten Teil tanzt sie zunächst inmitten der Tanzgruppe, weist aber dann die (ausgebildeten) TänzerInnen an, still zu halten und niederzuknien. Nun ist es an der Protagonistin, ihr Solo zu tanzen, während die anderen regungslos verharren. Anleihen zu Choreographien bekannter Popstars, die als SolokünstlerInnen souverän mit ihren Ensembles tanzen, sind hier unübersehbar. Neben dem direkten und basslastigen Musiksound von Banatuluis Titel Prin Straini kommt jetzt eine aufwendige Kameratechnik zum Einsatz. So werden die Tanzbewegungen Denisas vor allem durch Kranfahrten hervorgehoben. Die hohe Schnittfrequenz und Einstellungen aus der Vogelperspektive verorten die Sequenz im Bereich der Musikclip-Ästhetik (Keazro/Wübbena 2017). Deutlich wird dies besonders am Schluss, als sich Denisa mit geöffneten Armen um sich selbst dreht und die Kamera über ihrem Kopf ist und sich langsam von diesem entfernt und so die sich bewegende Protagonistin inmitten der anderen, im Stillstand befindlichen, TänzerInnen erscheinen lässt.

Der letzte Clip bildet den Höhepunkt und bewegt sich von der Siedlung in die Innenstadt hinein, wobei das Versprechen einer allumfassenden Tanz-Gemeinschaft weiter zugespitzt wird. Vor dem Clip zeigt eine Szene, wie die BewohnerInnen der Siedlung im Fernsehen einen Bericht über den Gerichtsprozess sehen, in dem über das Weiterbestehen der Siedlung entschieden wird. Denisa Gabor, so legt es der Film nahe, scheint die Situation - es geht um die Räumung der Siedlung nicht zu verstehen. Auf dem Soundtrack werden dumpfe Geräusche eingeblendet, die auf ihre Hörbehinderung verweisen. Der (filmische) Ausweg, der eröffnet wird, liegt wiederum in der Musik und in der Choreographie, in denen die Protagonistin nicht durch ihre Schwerhörigkeit behindert wird, ganz im Gegenteil: Sie wird wiederum zunächst allein in ihrer Hütte gezeigt. Mit grünem Tuch, gelbem Oberteil und diversen Accessoires bekleidet gibt sie energisch den Takt vor. Ihr Weg be- 
ginnt, so zeigt es der Clip, in der Siedlung, führt sie und die ihr folgenden Kinder aber rasch auf die bekannte Piasek-Brücke in der Stadt, auf der Kinder und Jugendliche aus der Siedlung und Wrocław nun gemeinsam tanzen. Es folgen Choreographien, in denen Denisa Gabor gemeinsam mit großen Gruppen von TänzerInnen agiert. SeniorInnen und weitere Passanten werden ebenfalls in das Geschehen einbezogen, das sich in den Gassen und Boulevards des historischen Zentrums, dem Salzmarkt mit unzähligen Blumenständen sowie auch dem Platz vor dem alten Rathaus in turbulentem Tempo und synchron zur Musik entfaltet. Hier wird eine Aneignung des Stadtraums nicht nur im Einklang mit deren BewohnerInnen tänzerisch inszeniert und filmisch im Rhythmus der Musik ins Bild gesetzt. Der Rhythmus der Stadt (Lefebvre 2004) wird außer Kraft gesetzt und mit einem anderen konfrontiert - „,der Rhythmus ist kritisch, er verknüpft kritische Momente, oder er verknüpft sich mit dem Übergang von einem Milieu in ein anderes. Er wirkt nicht in einem homogenen Zeitraum, sondern operiert mit heterogenen Blöcken" (Deleuze/Guattari 1987, S. 427). Zudem bewegt sich dieses Allinklusions-Szenario entlang wichtiger historischer Wahrzeichen der Stadt. Deren Status als originäre Elemente einer polnischen(-deutschen) Kultur und Geschichte wird durch die Choreographien und die Musik mit neuer Bedeutung aufgeladen. Nunmehr fungieren sie hier als Basis einer Vergemeinschaftungsidee, die keine klaren Grenzen mehr zwischen den Roma vor der Stadt und den BürgerInnen der Stadt einzieht. Dieses imaginierte Miteinander, das die Idee von Multikulturalität und Minderheitsdiskursen im urbanen Kontext der europäischen Union aufruft, wird auch von der letzten Einstellung betont: die Kamera setzt das Mädchen ins Zentrum, bewegt sich dann aber in einer Kranfahrt nach oben. Damit wird die Protagonistin, die sonst heraussticht, zu einem Farbtupfer unter den vielen anderen. Alle zusammen formen ein unregelmäßiges aber doch zusammengehörendes Muster, das den Rathausplatz bedeckt und von Kamera aus der Vogelperspektive sichtbar gemacht wird. Doch kurz darauf gibt es einen Szenenwechsel: Der Traum ist vorüber, wenn die nächste Einstellung ganz in grau getaucht (und etwas plakativ) zum Alltag der Protagonistin zurückkehrt und diese beim Bitten um Geld in jener Stadt zeigt, die gerade noch Schauplatz gemeinsamer Tanzaktionen war.

Durch den Tanz wird - so Zwiefka - die Protagonistin in die Lage versetzt, ihre Emotionen auszudrücken. Tanzen sei eine wichtige Praktik für sie, um sich durch ihre Bewegungen zum Rhythmus der Musik auszudrücken (Festival dei Popoli, 00:01:50). Der Film sei, wie eingangs erwähnt, ein Gemeinschaftsprojekt, das auf den Ideen Denisas aufbaue. Anhand der Tanzinszenierungen, für die von Zwiefka ein bekannter Choreograph und TänzerInnen engagiert wurden, lassen sich jedoch Spannungen bezüglich dieses ,partizipativen“ Ansatzes erkennen:

Can you imagine that we had like a scene with one hundred people dancing, rehearsed six weeks, you know, rehearsal, because Bollywood is all about synchronized dancing. And on the day we are shooting, starting at 6 am, Denisa says: No. I am not going to dance this. I mean she does not say it but she shows it. We had to change the choreography and the dancers were devastated. They said we have never done something like that in our entire lives. But this is her. She wants something and she goes for it. (Zwiefka in Katie chats) 
An diesem Zwischenfall wird ein Paradox offenbar: Wenn die Filmemacherin es als angebracht ansieht, die Choreografien in professionelle Hände zu geben und auf aufwendige Film-Technik zu setzen, so stellt dies für Denisa scheinbar eine Zumutung dar. Durch den Teilhabeprozess-Film entstehen Konflikte. Es ist die Protagonistin, die die Filmemacherin und ihre Pläne mit ihren Vorstellungen - soweit sie an dieser Stelle bekannt sind - konfrontiert und in eine andere Richtung drängt. "This is how tough she [Denisa] is, how strong she is and how she always fights for what she wants" (Festival dei Popoli, 00:05:25). Das sogenannte kollaborative Filmeschaffen ist folglich als ein Prozess zu begreifen, der gewisse Unwägbarkeiten mit sich bringt und eine Fähigkeit zur Improvisation verlangt: „Man bricht aus, wagt eine Improvisation. Aber improvisieren bedeutet, sich mit der Welt zu verbinden und zu vermischen“ (Deleuze/Guattari 1987, S. 425). In diesem filmischen Prozess generieren die eröffneten Möglichkeiten gemeinsamen Filmemachens um die Bollywood-inspirierten Tanzinszenierungen nicht nur Versprechen. Vielmehr sind es auch Zumutungen und Ungewissheiten, die diese spezifische Teilhabekonstellation (Bippus/Ochsner/Otto 2016) kennzeichnen.

Die verschiedenen Choreographien formulieren eine utopische Vision und ein Versprechen im Hinblick auf ein mögliches Zusammen-Leben der Menschen aus der Siedlung am Leben in der polnischen Stadt. Die Protagonistin wird als Hauptdarstellerin mit musikalischem Verve inszeniert, die den Takt angibt. In den so entworfenen Zwischenräumen werden jedoch auch Widersprüche deutlich. Diese entstehen etwa durch den - teils plakativ wirkenden - Kontrast zwischen bunten, schnell-geschnittenen Musikvideo-Sequenzen und anderen Teilen des Films, in denen eine beobachtender Modus vorherrscht und die Ins-Bild-Setzung der ,BettelÖkonomie' der Roma sowie die daran geknüpften Alltagshandlungen sich einer stereotypisierenden Sichtweise nicht ganz entziehen können. ${ }^{10}$ Des Weiteren birgt die Positionierung Denisa Gabors als Vorreiterin einer möglichen Inklusion der Roma weitere Fragen. Letztere stehen in Verbindung mit der populären RomaMusik von MusikerInnen wie Dani Printul Banatului, Dani Mocanu oder Irina Lepa, die in den Clips verwendet wird (Katie chats). Besonders auf Banatuluis und Lepas Youtube-Kanälen sind Musikvideos zu finden, die sich durch heteronormative Muster und sexistische Darstellungsweisen (vgl. Bonnette 2015, S.144; Sandve 2017, S. 170) charakterisieren. ${ }^{11}$ Vor diesem Hintergrund stellt sich der Effekt der Musikvideoszenen in The Queen of Silence als paradox dar: Einerseits wird die Protagonistin so überhöht dargestellt, dass sie als ein ,Aushängeschild‘ für ein Versprechen auf soziale Inklusion gilt. Ihre mehrfache Exklusion, herbeigeführt u.a. durch ihren Status als junge Frau, als Mensch mit Behinderung sowie als Migrantin, wird im Rahmen der Musikvideos als nahezu überwunden inszeniert. Ande-

$10 \mathrm{Zu}$ beachten ist jedoch, dass sowohl die Szenen im beobachtenden Modus als auch die „partizipativ“" angelegten Musikvideo-Choreografien jeweils als eigene, spezifische Teilhabekonstellation betrachtet werden müssen. In welcher Form erstere in The Queen of Silence entstanden, kann anhand des vorliegenden Materials hier nicht ausführlich beschrieben werden.

11 Auf die Lyrics kann an dieser Stelle auf Grund fehlender Sprachkenntnisse nicht eingegangen werden. 
rerseits lässt der Soundtrack an dieser Geste eines filmischen Empowerments (Bröckling) signifikante Risse entstehen, wird doch darin die Position der Frauen vor allem als sexualisiertes Objekt re-produziert. Es stellt sich die Frage, wie sich jene Denisa, die sich gegenüber Zwiefka und einem Filmteam sowie den TänzerInnen und dem Choreographen durchsetzt, auch gegenüber den paternalistischen Strukturen (nicht nur) ihrer Lebenswelt behaupten kann? Oder halten The Queen of Silence und ihre Protagonistin - diese „,very powerful young lady“ (Zwiefka in Festival dei Popoli, 00:01:35) - nicht doch durch die Konfrontation verschiedener Rhythmen dazu an, gerade überkommene geschlechterpolitische Ordnungen sowie auch marginalisierte Positionen von Menschen mit Behinderung zu überdenken? Die Effekte dieses filmisch gestalteten Zwischenraums sind ambivalent und lassen sich nicht ohne weiteres vereindeutigen.

\section{And-Ek Ghes...: Ein filmisch musikalisches Abenteuer der Familie Velcu in Berlin Reflexionen gemeinsamen Filmemachens}

Die Entstehung des Films And-Ek Ghes... geht auf eine Produktion Scheffners aus dem Jahr 2012 zurück: Revision ist ein Film über die Familien von Grigore Velcu und Eudache Calderar, deren Körper 1992 in Mecklenburg-Vorpommern an der deutsch-polnischen Grenze tot aufgefunden wurden. Beide wurden damals scheinbar bei dem Versuch erschossen, die europäische Außengrenze zu überschreiten. Der Fall sowie der damit verbundene Prozess gegen die verdächtigen Jäger, der mit einem Freispruch endete, wird von Scheffners Film aufgegriffen und in einer akribischen Recherchearbeit zusammen mit den rumänischen Familien aufgearbeitet. So entsteht ein „imaginärer filmischer Raum“ (Scheffner/Peters 2018, S. 72), der eine europäische Perspektive auf das Geschehene in einem Modus des Zweifelns entwirft (Reiter 2016). And-Ek Ghes... beginnt einige Zeit nach Revision, als Scheffner die Nachricht von Colorado Velcu (der Sohn von Grigore Velcu) erreicht, dass dieser mit seiner Familie aus Craiowa nach Deutschland kommt. In Essen trifft der Filmemacher auf die Familie und schenkt Velcus Tochter eine Digitalkamera. Filmtechniken bilden in der Folge eine Art der Kommunikation, die eine spezifische Form von Verständigung ermöglicht. Etwas später, als die Familie nach Berlin-Wedding zieht, entsteht die Idee zu einem Filmprojekt über das Leben der Velcus in Deutschland, über ihr Ankommen in diesem Land, ihre Probleme und Freuden im Alltag (00:11:41). Dabei ist Scheffner nicht der einzige, der die verschiedenen Situationen filmt. Vielmehr übernehmen die Familienmitglieder selbst die Regie und produzieren die Bewegtbilder über sich. Zentral ist dabei die Rolle von Colorado Velcu: Da seine Frau Teodora in Rumänien im Frauengefängnis in Targsor (Grigorovici 2014, S. 33) ist, ist er als alleinerziehender Vater von sieben Kindern auf sich gestellt. In dieser Situation gestaltet er den Film zusammen mit seinen Kindern und anderen Verwandten (Scheffner 2016). 
Einer der Ausgangspunkte für den Film sind Momente, in denen der filmende Scheffner selbst von der Familie gefilmt wird. Diese reflexive Situation wird zum Beginn einer Produktion von Bildern über das Leben der Velcus. Während des Produktionsprozesses wird Colorado Velcu zum Co-Regisseur. In dieser Hinsicht wehrt sich Scheffner gegen Behauptungen, dass er Velcu lediglich ,die Kamera gegeben“ oder ,überlassen` hätte (Jaeger 2016). Wie Scheffner erklärt:

Also für mich ist es vor allem ein Film über's Filmemachen und über die Möglichkeit, die hierarchischen Verhältnisse, die Film herstellt und die auch in And-Ek Ghes... benannt werden, für Momente zum Schwingen zu bringen. Die filmischen Möglichkeiten zu nutzen, um sie zu dehnen und sich zu positionieren, immer wieder neu zu positionieren, nicht als ein festgenageltes Subjekt da zu stehen. (Scheffner/Peters 2018, S. 77)

Scheffner legt dar, dass Velcu und er sich in regelmäßigen Abständen trafen, um das entstandene Material zu sichten und eine Auswahl daraus zu treffen. Dass es ein Gemeinschaftsprojekt der Familie - ohne ein im Vornherein festgelegtes Drehbuch - war, wird auch deutlich, wenn die unterschiedlichen verwendeten Bildformate von kleiner Digitalkamera bis Handy-Aufnahmen berücksichtigt werden. Wie es in einer Kritik zum Film heißt: „Die Digicams machen die Runde, jeder gibt seine Sicht der Dinge preis, erzählt irgendwo zwischen den Tatsachen und der Hoffnung auf ein besseres Leben“ (Bluth 2016). Insofern kann eine Multiplizierung von Blickpunkten ausgemacht werden, die daraus resultiert, dass die Gefilmten hier neben Scheffner auch als Filmschaffende positioniert werden, die gleichermaßen zur Entstehung der Produktion beitragen (vgl. Harper u.a. 2016, S. 27).

Von Beginn an ist der Film gegenüber seinen eigenen Bildern reflexiv und distanziert sich damit von diesen als authentisch gekennzeichneten Ins-Bild-Setzungen. Dies beginnt etwa mit einer Szene, in der Colorado Velcu in der Tonkabine sitzt, Scheffner aber nur flüchtig im Spiegelbild der Trennscheibe erkennbar wird. Velcu hört die Aufnahme seiner Stimme über seine Versuche Tagebuch zu schreiben in der voice over und beschließt nach Ende der Einspielung, dass der Take doch noch besser zu machen sei. Auch die Aufnahmen vom Einzug der Familie in die Essener Wohnung werden mehrfach gebrochen: Sie zeigen zum Beispiel, wie Scheffners Kamera-Beobachtungen der Familie in der neuen Berliner Wohnung auf einem Laptop vorgespielt werden. Die Velcus kommentieren die Bilder, wodurch mehrfach eine Art Situationskomik entsteht und zugleich die prekären Wohnverhältnisse ins Spiel gebracht werden. Die vielfachen Brüche und Distanzierungen initiieren einen beständigen Perspektivwechsel, durch den die Beteiligten in Beziehung zueinander gesetzt werden und als eine Art filmisches Kollektiv erscheinen. Es handelt sich um Teilhabebeziehungen zwischen tragbaren KameraTechnologien, Abspielgeräten sowie denjenigen, die die Bilder aufnehmen und sie anschauen. Darunter ist auch die Tochter Noami, die die Kamera geschenkt bekommen hat und nun ein Filmtagebuch führen kann - „um“, wie es Colorado Velcu an einer Stelle formuliert, ,schöne Erinnerungen festzuhalten“. Doch dies ist wiederum als ein situiertes Statement zu begreifen, das diesen ganz bestimmten 
Umständen, dem Moment der Ankunft und dem Blick in eine noch unbestimmte Zukunft geschuldet ist.

Die Bedeutung und das Konzept Familie werden mehrfach thematisiert. Dies geschieht in einer Weise, bei der Konventionen einer beobachtenden Kamera spielerisch aufgriffen werden. So zeigt eine Einstellung, aufgenommen von einer auf der Arbeitsfläche in der Küche positionierten Kamera, wie eines der Kinder für den älteren Bruder etwas zu trinken holen soll. Während die Kamera die fly-on-thewall-Position einnimmt und den müden Vater nach seinem Arbeitstag fokussiert, kommentiert dieser das Geschehen: „Immer kommandiert hier jemand herum. Geh vor, ich komme gleich und rede mit ihnen." Szenenwechsel ins Wohnzimmer, in dem vier Kinder auf dem Sofa sitzen und vom Vater zu hören bekommen, dass sie immer aufräumen sollen. In charmanter Weise wird das Material genutzt und gezeigt, wie Colorado Velcu ein Schmunzeln über die eigene Schauspielerei nicht verbergen kann. Dies wird anhand einer Regieanweisung von ihm noch unterstrichen: „Sitzt schön ruhig. So leise, ohne zu lachen, okay?“ Der Komik der Szene wird durch den Kommentar eines der Söhne Nachdruck verliehen: „Ich dachte schon, er redet wirklich so mit uns." Danach lachen alle. Auch das Ausschalten der Kamera, das erneut auf die Gemachtheit der Bilder verweist, ist im Film belassen. Solche Momente finden sich auch in einer Szene mit den drei Töchtern, die ihre Hausarbeiten erledigen. Die Regieanweisung Velcus lautet dort: „Nicht da hingucken. [Die Mädchen schauen in die Kamera.] Macht hier eure Sachen!“ Erst wenn er erneut ins Zimmer kommt, wird der zu filmende Dialog über die zu erledigenden Aufgaben zwischen Vater und den drei Töchtern in Szene gesetzt (00:14.3700:16:33). In der Sequenz wird die Kamera folglich dazu eingesetzt, ein Bild vom familiären Alltag der Velcus zu entwerfen. Vorstellungen von einem sensiblen, Tagebuch-schreibenden und alleinerziehenden sowie fürsorglichen Vaters werden entworfen (Scheffner/Peters 2018, S. 78). Doch die Art und Weise des Films mit dem Material umzugehen, legt eine Unbestimmtheit nahe und lässt so auch Zweifel aufkommen. Dies ist ganz im Sinne Scheffners, der meint: „Wie ein Vater sich als Familienvater darstellen möchte, finde ich politisch wesentlich brisanter und stärker, als irgendwie zu versuchen zu zeigen, wie die Familie denn tatsächlich funktioniert. Diesen Zweifel zu lassen: Machen die das jetzt extra für die Kamera? Dass sich Colorado und die Familie das herausnehmen, finde ich unheimlich befreiend“ (Scheffner in Jaeger 2016).

Die Herangehensweise an die Produktion des Films und der reflexive Umgang mit dem Material in der Postproduktion, an der Velcu und Scheffner beteiligt waren, ist in vieler Hinsicht interessant. Dies ist auch gerade deshalb der Fall, weil hier im Kontext einer Teilhabe an (zu erschaffenden) Filmbildern an einem Bild von migrierten Personen gearbeitet wird, dass nicht vorwiegend deren Probleme betont, obwohl die Schwierigkeiten - finanzielle Notlagen, Probleme mit Behörden wegen der Beschulung der Kinder etc. - auch benannt werden (Jaeger 2016): „Colorado und seine Familie haben keine Lust, lediglich als Opfer gesehen werden, sie verweigern sich dem klassischen Rollenbild des Migranten. Sie bewahren sich ihre Würde, ohne die Lage dabei jemals zu verklären“ (Barwenczik 2016). Dies wird 
über den Film vermittelt, der hier in seiner umfassenden strukturellen, sozialen und technologischen Dimension also zu einem Mediator wird, innerhalb dessen und durch den sich Deutungsmöglichkeiten eröffnen und verteilte Handlungsinitiativen beobachtbar gemacht wird. So wird eine schematische Sichtweise auf „die Roma“ (vgl. Hagen 2009; Bogdal 2011) in ein neues Licht gesetzt: „Es gibt eine große Gefahr, gerade in einem Film, der wirklich zufälligerweise eine Roma-Familie als Sujet hat, dass dann alle möglichen Authentizitätsbegriffe abgerufen werden: So ist die Roma-Familie an sich. Genau das ist das Problem, dass es unheimlich schwierig ist, eine Familie als Familie zu sehen und erst in zweiter Linie als RomaFamilie. Das ist das, was der Film versucht" (Scheffner in Jaeger 2016).

\section{Musikalisches Selfie vor dem Brandenburger Tor}

Obwohl es nicht vordergründig auf der Hand liegen mag, so spielt doch Musik und Gesang eine wichtige Rolle in And-Ek Ghes.... Mit welchen Bedeutungen diese Verknüpfung von Sound, Körpern und Film hier versehen wird, und welche Konsequenzen dies für den Film als eine Teilhabekonstellation hat, wird der folgende Absatz herausarbeiten. Eine erste musikalische Performance von Parizan Nistor aka „Parry“'12 ist Teil der Sequenz, die die Ankunft der Familie in Berlin und die ersten Ideen über den zu realisierenden Film behandelt. Versatzstückartig und fragmentarisch zeigt der Film einige Einstellungen von asiatischen KampfsportÜbungen, Boxtraining zu Hause, Verfolgungsjagden auf dem Spielplatz. Bezug genommen wird nicht nur auf Action-Genres, sondern auch auf romantische Beziehungsdramen. Dazu zählt die Szene mit der Gesangseinlage Parrys ${ }^{13}$ : Dieser streift mit seinem Cousin durch Berlin, tut aber so, als würden sie sich in London aufhalten, wie aus dem Dialog hervorgeht. Dabei bittet ihn der Cousin, ein Lied für seine Geliebte zu singen. Parizan stimmt sogleich eines an, während er die Kamera hält und die menschenleere Allee mit dem Cousin zeigt. Die Performance ist im Handy-Hochformat gefilmt und nicht geschnittenen. Hier wird durch den Rückgriff auf eine Form des Cellphilms (McEntee 2016) nicht nur ein Bezug zu gegenwärtiger digitaler mobiler Jugendkultur herstellt. Die verwackelten Handybilder muten zudem wie ein Spielfilm-Fragment an: verliebter Junge wandert gedankenversunken durch die Stadt, während seine Gedanken bei seiner Geliebten sind. Es entsteht eine sentimentale Stimmung, die durch die Gesangsmelodie aus dem Off betont wird (00:12:35-00:13:59). Die zweite Gesangsperformance ereignet sich am Rande eines Picknicks im Park in einer Szene mit Raţa Miclescu und Parizan Nistor. Letzterer gibt ihr die Kamera und fordert sie auf ihn zu filmen, da er das „traditionelle“ Roma-Lied Gelem Gelem singen wird. Diesem bekannten Roma-Lied kommt hier

12 In den Unterlagen des Films wird Parizan Nestor oder Parry sowie auch Parica genannt. Vgl. https://andekghes.pong-berlin.de/ (Zugriff am 29 Oktober 2018).

13 Um Missverständnisse zu vermeiden, wird der Sohn Colorado Velcus im Folgenden mit dem Vornamen genannt. 
eher eine emotionale, denn politische Konnotation zu. ${ }^{14}$ Die romantische Facette wird nicht zuletzt dadurch herausgestellt, dass sich beide in dieser Situation vom Picknick entfernt haben und zu zweit auf der Terrasse eines Schnellimbiss verbringen (ohne dass sie sich dabei näher kommen). Wichtig indes zu bemerken, dass technologische Aspekte der Aufnahmesituation wiederum über die Tonspur vermittelt werden. Dort fragt die junge Frau, ob sie den „roten Knopf“ drücken müsse, was das hier technik-affin wirkende und singende Gegenüber verneint, da die Aufnahme bereits läuft. Demnach werden hier auch Geschlechterrollen verhandelt, wobei der Filmende ihr zwar die Kamera überlässt, aber doch die Kontrolle über die Situation behält. Jedoch ist es dann Raţa, die das Hochformat verlässt und das Handy in die horizontale Position dreht. Die Einstellung betont wiederum die Spontaneität der Performance und ruft dabei eine Standard-Filmsituation auf, in der die Protagonistin vom Verehrer ein „Ständchen“" gesungen bekommt.

Die musikalischen Ambitionen von Colorado Velcu und seinem Sohn kulminieren in einem Musikclip am Ende des Films. ${ }^{15}$ In einer langen Einstellung erklärt Velcu zuvor, wie das selbst komponierte Lied And-Ek Ghes... entstand. Es sei eine Geschichte, wie sie oft bei den Menschen aus Faţa Luncii, dem Stadtteil des rumänischen Craiovas mit einem hohen Roma-Anteil, vorkommen würde. Sie sei aber auch von seiner eigenen Geschichte inspiriert und ein Lied über ,die Liebe zwischen zwei jungen Leuten“, bei der ein junger Mann um seine Geliebte kämpft und sie heiraten möchte, obwohl ihre Familie dagegen ist (01:14:54-01:15:29). Dem Clip gehen Szenen voraus, die Creatia Velcu im spanischen Badalona bei einem Tanzwettbewerb zeigen und von ihrem Wunsch erzählen, ein Star zu werden. Parizan wird hingegen bei der Gesangsprobe in einem Tonstudio gefilmt. Colorado Velcu kommt dann als Berliner Handwerker ins Bild, der von der Baustelle in ein Gebäude gelangt und dort anfängt Orgel zu spielen. Somit ist der Start für das Musikvideo gegeben, der durch eine nunmehr künstliche Beleuchtung des Settings angezeigt wird. In diesem erscheinen der Sänger „Parry“ und seine Geliebte, gespielt von Creația Velcu. Dokumentarische Register und Ästhetik des Musikvideos werden hier miteinander in Resonanz gebracht: „Das [Musikvideo am Ende] ist auch ein Widerstand gegenüber bestehenden Bildpolitiken. Am Ende wurde es immer mehr zum Spielfilm. Es geht hier wirklich überhaupt nicht um Authentizität. Das war allen bewusst und auch der Spaß daran" (Scheffner in Jaeger 2016).

Der Clip mit dem Titelsong des Films entwirft eine Art Rundreise durch die deutsche Hauptstadt, wobei die wichtigsten touristischen Attraktionen wie Berliner

14 Die Melodie des Lieds war unter den Roma auf dem Balkan weit verbreitet und wurde in den 1960er Jahren durch den Film I even met happy gypsies (1967, Jugoslawien) bekannt. 1971 wurde das Lied mit einem Text von Jarko Jovanovic versehen und als offizielle Hymne auf dem 1. World Romani Congress in London gesungen. In dieser Form hat der Text eine starke politische Bedeutung, da er sich auf den Porajmos, d.h. auf die Ermordung der Sinti und Roma durch das nationalsozialistische Regime bezieht (Hemetek 2017, S. 96-101).

15 Dass es ein eigener Clip ist zeigt der Umstand, dass das Video auch als extra Post vom Nutzer P. Scheffner am 02.02.2016 auf youtube eingestellt wurde. Vgl. https://www.youtube.com/watch? $\mathrm{v}=\mathrm{mV} 9 \mathrm{G} 2 \mathrm{G} 5$ bjrc (Zugriff am 12 Januar 2018). 
Fernsehturm, Museumsinsel, Berliner Dom, die Spree mit ihren Touristendampfern ins Bild gesetzt werden. Die ProtagonistInnen werden in wechselnden farbenfrohen Outfits an den unterschiedlichen Plätzen der Stadt gezeigt. Es ist eine romantische Story, die die Liebe der Beiden zueinander, deren Konflikte mit den Eltern und den Start in ein Berliner Leben inszeniert. Diese klassisch gestrickte Erzählung bedient sich konventioneller Ästhetik und heterosexueller Geschlechterverteilungen. Das Bild der Stadt fügt sich fragmentarisch und sprunghaft durch die filmischen Versatzstücke zusammen. Wichtig ist hier der Umstand, dass die Schauplätze nicht im Wedding liegen, in dem die Familie wohnt, sondern zu den bekannten Attraktionen des Innenstadtbereichs zählen. Somit werden die Romanze der ProtagonistInnen und ihr „Kampf um eine gemeinsame Zukunft“ (Making Of) im Zentrum der deutschen Hauptstadt angesiedelt. Diese Kulisse großstädtischer Hotspots wird in AndEk Ghes... zudem durch Bollywood-Rhythmen in Schwingung versetzt (vgl. Barwenczik 2016). In diesen Klängen verknüpfen sich die Kompositionen Velcus mit dem Arrangement Vivek Philips und der Performance von MusikerInnen aus Goa (Making Of, BollywoodMDB). Ähnlich wie in The Queen of Silence macht sich hier eine Bewegung bemerk- und hörbar, mit der der Film den ProtagonistInnen eine ihnen scheinbar nicht zugängliche Sphäre eröffnen möchte. Teilhabe erscheint als ein Versprechen und (utopischer) Versuch, den nationalen Symbolen sowie deren Rhythmus in diesem filmischen Zwischenraum eine anders akzentuierte Klangfarbe zu verleihen.

Ein Höhepunkt der Klang-Odyssee durch die Hauptstadt ist ein Selfie des Liebespaars vor dem Brandenburger Tor, das auch als Postermotiv für die Ankündigung des Films genutzt wurde. Mit dieser Ins-Bild-Setzung werden nicht nur jugendkulturelle oder touristische Praktiken aufgerufen. Ähnlich wie bei den bereits erwähnten Handy-Filmen wird eine Verbindung, wenn nicht gar Verwandtschaft von migrantischen Praktiken und Praktiken der Mehrheitsgesellschaft nahegelegt. Durch den Clip werden unterschiedliche Elemente miteinander gekoppelt: filmische und musikalische Gestaltungsmittel, die Orte der Stadt und die Performance der ProtagonistInnen sowie digitale Medientechnologien. Das (Sound-)Selfie erweist sich somit als eine - auch migrantische - Selbst-Technologie (vgl. Otto 2017; Plohr/Otto 2015, S. 28). Es schlägt darüber hinaus eine filmisch-räumliche Situierung vor, die den Start in eine - hoffnungsvolle - Zukunft mit und in Berlin signalisiert und damit auch eine spezifische mediale Teilhabekonstellation der gerade in Deutschland angekommenen Velcus problematisiert:

Mit dem klassischen wohlmeinenden Dokumentarfilmblick denkt man, die haben sicher ganz viele Probleme mit der deutschen Bürokratie, weil man ja selber viele Probleme mit ihr hat. Und natürlich ist das auch so. Colorado würde aber nie auf die Idee kommen, im Jobcenter eine Kamera anzumachen. Das Beharren darauf, dass die Familie bestimmte Bilder bekommt, das hat für mich einen politischen Gestus. Das kulminiert dann im Musikvideo: Das sind Bilder, die man mit Berlin verbindet, aber nicht unbedingt mit einer Roma-Familie. Warum gibt es keine Bilder von Roma-Familien, wie sie sich vorm Brandenburger Tor fotografieren? Sie machen das alle! Da haben wir entschieden, ganz bewusst nach der Formel von Bollywood eine Wiederaneignung von Berlin 
zu vollziehen und diesen Bildern die gleiche Bedeutung zu geben wie anderen.“ (Scheffner, zit. n. Jaeger 2016, o.S.)

Auch dieser filmpolitische Schachzug, der zugleich als populärkulturelle Zumutung für einen Regisseur wie Scheffner verstanden werden kann, ${ }^{16}$ bleibt jedoch nicht unkommentiert. Denn zum Ende des Clips findet sich das Liebespaar im Sony Center am Potsdamer Platz und sukzessiv im Kino des Arsenals. Institut für Film und Videokunst e.V. wieder, in dem Om Shanti Om (2007) aufgeführt wird. ${ }^{17}$ Das romantische Abenteuer endet folglich mit einem Bollywood-Kinoabend, bei dem auch Colorado Velcu als Filmvorführer nicht fehlen darf. In diesem Sinne, so liegt zu vermuten, verortet der Musikclip die Herausforderung, sich mit Popkultur zu beschäftigen, gerade auch an einem Ort intellektueller Auseinandersetzungen mit dem Medium Film. Die Konfrontation dieser heterogenen Rhythmen wirft mithin die Frage auf, wie ,die Blickverhältnisse - die Machtverhältnisse, die sich in Blicken niederschlagen -, das Sprechen über und das Sprechen für, ins Schwingen zu bringen [sind]“" (Scheffner/Peters 2018, S. 82).

\section{Schluss: Audiovisuelle Teilhabe-Konstellationen als situierte Wissenspraktiken und kosmopolitisches Projekt}

Der Umgang mit abseitigen, illegal(isiert)en oder innenstädtischen Szenerien sowie migrantischen ProtagonistInnen und deren Praktiken erweist sich in beiden Filmen als zentraler Bestandteil einer Auseinandersetzung mit marginal(isiert)en Lebenswirklichkeiten. Diese dokumentarischen Produktionen werden zudem durch Musik und den Einsatz einer Musikvideoästhetik als audiovisuelle Teilhabekonstellationen beobachtbar und beschreibbar. Die Arbeit von Zwiefka lässt sich am ehesten in einem Bereich der Shared Anthropology à la Jean Rouch verorten (Ginsberg 1995; Harper u.a. 2016, S. 19). Der kollaborative Ansatz wird dort als Zusammenarbeit von EthnografInnen mit sozialen Gruppen verstanden (vgl. Harper u.a. 2016,

16 Viele KritikerInnen, die eine theoretische Auseinandersetzung mit Scheffners Havarie verfolgen, fanden es verwunderlich, dass dieser künstlerisch anspruchsvolle Regisseur And-Ek Ghes... realisiert hat: „Wie Havarie in der Kunst- und Theoriewelt sehr stark wahrgenommen wurde, darüber freue ich mich. Von And-Ek Ghes... waren alle etwas verwirrt - wieso jetzt Bollywood? Aber es geht wirklich um etwas sehr, sehr ähnliches [...]. In beiden Projekten geht es grundsätzlich, um diese Frage, wie Augenhöhe möglich ist. [...] Das ist in beiden Filmen, mit sehr unterschiedlichen Mitteln, der Ansatz“" (Scheffner/Peters 2018, S. 82).

17 Diese Konstellation treibt das Spiel mit dem Zweifel an der dokumentarischen Repräsentation und filmischen Wahrheit (Steyerl 2008) gewissermaßen auf die Spitze. Denn damit werden nicht nur Bezüge zur 58. Berlinale von 2008 aufgerufen, auf der Om Shanti Om unter Anwesenheit von Shahrukh Khan dem Publikum präsentiert wurde. Auch die reflexive Dimension der Spielfilmproduktion (Ciecko 2016; Gehlawat 2015, S.3; Sarkar 2013; Shastri 2011), in dem die Filmgeschichte Bollywoods in komplexer Weise zum Thema wird, kommt somit in And-Ek Ghes... nunmehr auf eine andere Ebene transponiert - zum Vorschein. So scheint die Produktion auf die Unabschließbarkeit der Befragung und Gestaltung einer wie auch immer gearteten (Film-)Wirklichkeit (Hohenberger 2006, S. 25-28) zu verweisen. 
S. 20). Die ProtagonistInnen werden dann wieder einbezogen, wenn der Film fertig geschnitten ist und sie ihn von der Regisseurin präsentiert bekommen. Insofern hat The Queen of Silence den Anspruch, zwischen Sinti und Roma sowie den BürgerInnen der polnischen Stadt zu vermitteln, ziviles Engagement zu zeigen und soziale Probleme zu diskutieren. Das Filmprojekt versucht dies im Einklang mit den Ersteren. Zwiefka entwickelte ein Vertrauensverhältnis mit den Sinti und Roma, um den Rhythmus der Siedlung zu verstehen und eine andere Repräsentationspolitik zu entwerfen - obgleich der Film (paradoxerweise) bestimmte Stereotypisierungen trotzdem aufruft. Die offene Frage bleibt, wie und in welchem Maße die Zusammenarbeit hinsichtlich des Films zwischen Zwiefka und Denisa aussah. Denn dies wird nicht im Film selbst reflektiert. Allerdings wird in Bezug auf die Entstehungsprozesse deutlich, dass asymmetrische Machtrelationen auch in ,partizipativen“ Ansätzen nicht verschwinden (Harper u. a. 2016, S. 20-22). Die Konflikte betrafen u.a. die Planung und Realisierung der Choreographien, werden aber im Film nicht weiter thematisiert. Dies zu tun wäre sicher eine Herausforderung gewesen. Doch hätte eine solche Reflexion die Möglichkeit eröffnet, etwa die Präsenz der Kamera in dem sozialen Gefüge zu problematisieren oder gegebenenfalls die ästhetisierenden Einstellungen, mit denen die Siedlung mehrfach in den Blick genommen wird, zu brechen. Denn, wie Donna Haraway bemerkt: „Im Anspruch, eine Perspektive aus der Position der weniger Mächtigen einzunehmen, liegt allerdings auch die ernst zu nehmende Gefahr einer Romantisierung und/oder Aneignung dieser Sichtweise. Das Sehen von unten ist weder einfach zu lernen noch unproblematisch [...]" (Haraway 1996, S. 228). Nichtsdestotrotz zählt The Queen of Silence zu jenen Darstellungen, die ,angemessenere, nachhaltigere, objektivere, transformierendere Darstellungen der Welt" (ebd.) formulieren möchten.

In Scheffners Film multiplizieren sich die Standpunkte anhand der verschiedenen eingesetzten Aufnahmen, wodurch auch die Produktion der filmischen Wirklichkeit selbst zum Gegenstand der Auseinandersetzung wird. Deren Bedeutung erweist sich somit im Sinne Minh-Has als politisch: „Bedeutung kann deshalb nur dann politisch sein, wenn sie nicht so leicht festzuschreiben ist und sich nicht nur auf eine einzige Quelle oder Autorität verlässt, sondern sie im Gegenteil entleert oder dezentralisiert" (Minh-Ha 2006, S.286). Die Vorgehensweise, die And-Ek Ghes... durch seine Machart beobachtbar macht, reflektiert und kritisiert somit essentialistische Repräsentationspraktiken, deren widersprechender Gestus sich oft als ambivalent erweist, indem er bekannte Bilder aufrufen muss, um diese zu verurteilen. Damit wird zugleich das Problem einer „Sicht von unten“, jene der „Unterworfenen“ adressiert, das schon Haraway in ihrer Diskussion des „situierten Wissens“ beschäftigt hat: „Aber das Problem, wie von unten aus zu sehen sei, erfordert mindestens ebensoviel Geschick im Umgang mit Körpern und Sprache und mit den Vermittlungen der Vision wie die hochentwickeltsten technowissenschaftlichen Visualisierungen" (Haraway 1996, S. 228, Hervorhebung im Orig.). Ein solches Geschick scheint sich in And-Ek Ghes... zwischen Aufnahmen von Handys, portablen Digitalkameras, den ProtagonistInnen und ihren Verortungen in der Wohnung im Wedding, im Park oder dem Berliner Stadtzentrum zu entspannen. Die 
Sicht auf die Welt und ihre Zwischenräume bzw. noch eher ihre bedeutungsvolle Produktion im Rahmen des Films wird gerade durch den Rückgriff auf eine „partiale Perspektive“ (Haraway 1996, S.226) ermöglicht. Nicht die Roma-Familie oder die MigrantInnen werden dargestellt, vielmehr macht die Produktion „eine Vielfalt partialen, verortbaren, kritischen Wissens [beobachtbar], das die Möglichkeit von Netzwerken aufrechterhält" (ebd., S. 229). Gewissermaßen kann man sagen, dass der Film den Versuch verfolgt, „eine kritische Praxis zur Wahrnehmung unserer eigenen bedeutungserzeugenden, ,semiotischen Technologien' zu entwickeln [...]" (ebd., S. 223). In diesem Sinne wirft die Produktion mehr Fragen auf, als er Antworten zu geben sucht. And-Ek Ghes... nährt einen steten Zweifel an jenen durch ihn selbst produzierten Wirklichkeiten (vgl. Reiter 2016, S. 105). Indem Scheffner und seine ProtagonistInnen mit audiovisuellen Technologien eine filmische „Ökologie der Praktiken“ (Stengers 2009) beobachtbar machen und reflektieren, scheinen sie ein kosmopolitisches Unterfangen im Sinne Stengers zu verfolgen. Diesem ,geht es um die Erfindung riskanter Wissenspraktiken, die Gemeinsames und Trennendes hervorbringen, die spekulativ und konstruktiv sind, die Unvernommenes vernehmbar machen und Unsichtbares sichtbar" (Harrasser 2013, S. 253). Das kontinuierliche Brechen von Sicherheiten einer ZuschauerInnenposition, die sich gerade eingerichtet hat, um einer Erzählung über kontemporäre Marginalisierungen beizuwohnen, wie sie von And-Ek Ghes... realisiert wird, kann folglich in einer Verwandtschaft zu Stengers (2005) Vorschlag gesehen werden, der „die Verlangsamung politischer Prozesse durch Miteinbeziehung wirklicher und möglicher Konsequenzen“" (Harrasser 2013, S. 243) fordert.

Die beiden Produktionen gewähren folglich auf unterschiedliche und vielfältige Weise einen Eindruck, von den Verknüpfungsmöglichkeiten filmischer Praktiken, Musik und städtischer Orte. Insbesondere die (imaginierten) Teilhabebeziehungen, die mit Blick auf die Musikclips und die ProtagonistInnen untersucht wurden, verweisen auf die Möglichkeit, Zwischenräume zu gestalten und gegenwärtigen gesellschaftlichen Konstellationen akzentuierte Rhythmen zu verleihen. Dabei erhalten die adressierten Schauplätze mitunter neue Klangfarben. Den ProtagonistInnen bieten sich bislang noch selten besetzte Rollen an. Die Bedeutungen von Filmtechnologien multiplizieren sich. Prozesse von Einschluss und Ausschluss werden problematisiert, reflektiert sowie mitunter auch durch spezifische Grooves nachvollziehbar. Doch bleibt der Ausgang ungewiss: Es eröffnet sich vielmehr ein resonanzfähiger Horizont, um dessen Linie/n herum Bedeutungen zu zirkulieren beginnen, sonische Körper erscheinen, Film-Bilder sich miteinander verknüpfen und mediale Teilhabe an Kontur gewinnt. 


\section{Literatur}

Athanasiu, A./Butler, J. (2014): Die Macht der Enteigneten. Das Performative im Politischen. Zürich: Diaphanes.

Barwenczik, L. (2016): And-Ek Ghes von Colorado Velcu und Philip Scheffner, Kino-Zeit. In: KinoZeit.de Arthouse - Film \& Kino, http://www.kino-zeit.de/blog/berlinale-2016/and-ek-ghes-voncolorado-velcu-und-philip-scheffner (Zugriff am 20. Dezember 2017).

Bergermann, U. (2003): Videoclip. In: Hügel, H.-O. (Hg.): Handbuch Populäre Kultur: Begriffe, Theorien und Diskussionen. Stuttgart: J.B. Metzler, S. 478-482.

Bippus, E./Ochsner, B./Otto, I. (2015): Between Demand and Entitlement: Perspectives on Researching Media and Participation. In: Denecke, M. u.a. (Hg.): ReClaiming Participation. Technology, Mediation, Collectivity. Bielefeld: transcript, S. 261-286.

Blecking, D. (2014): „Wie sollt ihr leben, hier auf dieser Welt“. Die polnischen Roma zwischen Tradition und Aufbruch. In: Dossier „Sinti und Roma“. Bundeszentrale für politische Bildung, http:// www.bpb.de/internationales/europa/sinti-und-roma-in-europa/197233/die-polnischen-roma-zwi schen-tradition-und-aufbruch-wie-sollt-ihr-leben-hier-auf-dieser-welt?p=all (Zugriff am 20. Dezember 2017).

Bluth, J. (2016): And-Ek Ghes... Kritik. In: Critic 21.09. http://www.critic.de/film/and-ek-ghes9355/ (Zugriff am 17. Januar 2018).

Bogdal, K-M. (2011): Europa erfindet die Zigeuner. Eine Geschichte von Faszination und Verachtung. Berlin: Suhrkamp.

Bollywood Movie Data Base: Vivek Philip. Music Director. http://www.bollywoodmdb.com/celebri ties/filmography/vivek-philip/4179 (Zugriff am 12. Januar 2018).

Bonnette, L. M. (2015): Pulse of the People: Political Rap Music and Black Politics. Philadelphia, Pa: University of Pennsylvania Press.

Bröckling, U. (2003): You are not responsible for being down, but you are responsible for getting up. Über Empowerment. In: Leviathan 31 (3), S. 323-344.

Brunner, A. (2017): Balkanmusik auf dem westeuropäischen World Music-Markt. In: Leggewie C., Meyer E. (Hg.): Global Pop. Das Buch zur Weltmusik. Stuttgart: Metzler, S. 276-282.

Bunescu, I. (2014): Roma in Europe the politics of collective identity formation. Farnham: Ashgate.

Chion, M. (1994): Audio-vision: Sound on screen. New York: Columbia University Press.

Ciecko, A.(2016): Reflexive Global Bollywood and Metacinematic Gender Politics in Om Shanti Om (2007), Luck By Chance (2008), and Dhobi Ghat (2010). In: Diogenes 62 (1), S. 24-37.

Coleman, G. (2017): Romani Musicians. The Fantasy of the Exotic in Film and Popular Culture. Bachelor Thesis, International Studies, University of Iowa.

Deleuze, G./Guattari, F. (1987): Tausend Plateaus. Berlin: Merve.

Dobreva N. (2007): Constructing the 'Celluloid Gypsy'. Tony Gatlif and Emir Kusturica's 'Gypsy films' in the context of New Europe. In: Romani Studies 17 (2), S. 141-153.

Fings, K. (2016): Sinti und Roma Geschichte einer Minderheit. München: C.H. Beck.

Friedner, M.I./Helmreich, S. (2012): Sound Studies Meets Deaf Studies. In: The Senses and Society 7 (1), S. 72-86.

Gehlawat, A. (2015): Twenty-First Century Bollywood. London: Routledge.

Grigorovici, M. (2014): Institutionalizing the Intersectionality between Gender and Ethnicity in Romania: The Case of Roma Women, Master Thesis. Faculty of Law. Lund University, http://lup. lub.lu.se/luur/download?func=downloadFile\&recordOId=4538660\&fileOId=4647711 (Zugriff am 17. Januar 2018).

Hagen, K. (2009): Inszenierte Alterität. Zigeunerfiguren in Literatur, Oper und Film. München: Fink.

Haraway, D. (1996): Situiertes Wissen. Die Wissenschaftsfrage im Feminismus und das Privileg einer partialen Perspektive. In: Scheich, E. (Hg.): Vermittelte Weiblichkeit. Feministische Wissenschafts- und Gesellschaftstheorie. Hamburg: Hamburger Edition, S. 217-248. 
Harrasser, K. (2013): Treue zum Problem. Situiertes Wissen als Kosmopolitik. In: Deuber-Mankowsky, A./Holzhey, Ch. F. E. (Hg.), Situiertes Wissen und regionale Epistemologie. Zur Aktualität Georges Canguilhems und Donna J. Haraways. Wien: Turia + Kant, S. 241-259.

Harrasser, K. (Hg.) (2017): Auf Tuchfühlung. Eine Wissensgeschichte des Tastsinns. Frankfurt, New York: Campus.

Hemetek, U. (2017): Roma and "their" Music in South-eastern Europe: Silenced Voices? Exclusion, Racism and Counter-Strategies. In: Kirkegaard, A./Järviluoma, H./Sverre Knudsen, J. (Hg.): Researching Music Censorship. Cambridge Scholars, S. 83-103.

Henriques, J. (2011): Sonic bodies. Reggae sound systems, performance techniques, and ways of knowing. New York, NY: Continuum.

Hohenberger, E. (2006): Dokumentarfilmtheorie. Ein historischer Überblick über Ansätze und Probleme. In: Dies. (Hg.): Bilder des Wirklichen. Texte zur Theorie des Dokumentarfilms. Berlin: Vorwerk 8, S. 9-33.

Jaeger, F. (2016): Regisseur Philip Scheffner über Roma-Doku. Ab hier übernimmt Colorados Clan. In: Spiegel Online 25.09., http://www.spiegel.de/kultur/kino/philip-scheffner-dokumentarfilmeueber-roma-und-ein-fluechtlingsboot-a-1113219.html (Zugriff am 17. Januar 2018).

Joncheere, A./Vandevelde, I. (2016): Representing Rajasthani roots: Indian Gypsy identity and origins in documentary films. In: Romani Studies 26 (2), S. 151-173

Keazor, H./Wübbena, T. (2017): Musikvideo. In: Hecken, T./Kleiner, M. S. (Hg.): Handbuch Popkultur. Stuttgart: J.B. Metzler, S. 173-177.

Klöß, S. (2014): Notting Hill Carnival. Die Aushandlung des Eigenen im multiethnischen Großbritannien seit 1958. Frankfurt/M.: Campus.

Koch, U. (2010): Soziale Konstruktion und Diskriminierung von Sinti und Roma. In: Hormel, U./ Scherr, A. (Hg.): Diskriminierung: Grundlagen und Forschungsergebnisse. Wiesbaden: VS Verlag für Sozialwissenschaften, S. 255-278.

Lefebvre, H. (2004): Rhythmanalysis. Space, time and everyday life. London: Continuum.

Macentee, K./Burkholder, C./Schwab-Cartas, J. (2016): What's a Cellphilm? An Introduction. In: Dies. (Hg.): What's a Cellphilm? Integrating Mobile Phone Technology into Participatory Visual Research and Activism. Rotterdam: SensePublishers, S. 1-15.

Making Of. And-Ek Ghes... Der Song. Pong Berlin, http://andekghes.pong-berlin.de/de/10/and-ekghes-der-song (Zugriff am 18 Januar 2018).

Marshall, C. (2014): Crippled Speech. In: Postmodern Culture 24 (3), doi:10.1353/pmc.2014.0020 (Zugriff am 17. Januar 2018).

Milne, E-J./Mitchell, C./De Lange, N. (Hg.) (2012): Handbook of participatory video. Lanham: AltaMira Press.

Otto, I. (2017): Migrantische Gemeinschaftlichkeit und mobile Medien. Habilitationsvortrag. 17.05. Universität Konstanz.

Pasqualino C. (2008): The Gypsies, Poor but Happy. In: Third Text 22 (3), S. 337-345.

Plohr, N./Otto, I. (2015): Selfie-Technologie. In: Pop. Kultur und Kritik 4 (1), S. 26-30.

Procter, J. (2003): Dwelling places. Postwar black British writing. Manchester: Manchester University Press.

Rancière, J. (2006): Die Aufteilung des Sinnlichen. Die Politik der Kunst und ihre Paradoxien. Berlin: b_books.

Reiter, L. (2016): Revisionen. Ästhetik des Anfangens und verkörperte Kritik. In: SYN. Magazin für Theater-, Film- und Medienwissenschaft 12, S. 101-109.

Rogers, M. (2016): Problematising participatory video with youth in Canada: the intersection of therapeutic, deficit and individualising discourses. In: Area 48 (4), S. 427-434.

Ruby, J. A. Y. (1992): Speaking For, Speaking About, Speaking With, or Speaking Alongside - An Anthropological and Documentary Dilemma. In: Journal of Film and Video 44 (1/2), S. 42-66.

Sandve, B. (2017): Staging the 'street boy'. Transculturalism, realness and hypermasculinity in the Norwegian rapper Jesse Jones. In: Hawkins, S. (Hg.): The Routledge research companion to popular music and gender. London: Routledge, S. 166-181. 
Sarkar, B. (2013) Metafiguring Bollywood: Brecht after Om Shanti Om. In: Sen, M./Basu, A. (Hg.): Figurations in Indian Film. London: Palgrave Macmillan, S. 205-235.

Scheffner, P. (2016): Ich filme dich, und du filmst mich. In: Pong Film, http://andekghes.pong-berlin. de/de/10/philip-scheffner (Zugriff am 17. Januar 2018).

Scheffner, P./Peters, K. (2018): Auf Augenhöhe arbeiten. Philip Scheffner im Gespräch mit Kathrin Peters. In: Busch, K. u.a. (Hg.): Wessen Wissen? Situiertheit und Materialität der Künste. Paderborn: Fink, S. 69-88.

Shastri, S. (2011) „The play's the thing, wherein I'll catch the conscience of the king”: Intertextuality in Om Shanti Om. In: Journal of Film and Video 63 (1), S. 32-43.

Silverman, C. (2012): Romani Routes. Cultural Politics and Balkan Music in Diaspora. Oxford: Oxford University Press.

Stengers, I. (2005): The Cosmopolitical Proposal. In: Latour, B./Weibel, P. (Hg): Making Things Public. Cambridge: MIT Press, S. 994-1003.

Stengers, I. (2009): Ökologien. In: Zeitschrift für Kulturwissenschaften 2, S. 29-34.

Steyerl, H. (2008): Die Farbe der Wahrheit. Dokumentarismen im Kunstfeld. Wien: Turia + Kant.

Sudbury, S. (2016): Locating a 'third voice': participatory filmmaking and the everyday in rural India. In: Journal of Media Practice 17 (2-3), S. 213-231.

Szeman, I. (2009): "Gypsy Music" and Deejays: Orientalism, Balkanism, and Romani Musicians. In: The Drama Review 53 (3), S. 98-116.

Trauschein, T. (2014): Die soziale Situation jugendlicher „Sinti und Roma“. Wiesbaden: Springer VS.

Turetzky, P. (2002): Rhythm: Assemblage and Event. In: Strategies: Journal of Theory, Culture \& Politics 15 (1), S. 121-138.

Tzanelli, R. (2016): Mobility, modernity and the slum the real and virtual journeys of "Slumdog Millionaire". London New York, NY: Routledge.

Uhlig, A. (2012): Ethnographie der Gehörlosen: Kultur - Kommunikation - Gemeinschaft. Bielefeld: transcript.

Waldschmidt, A. (2015): Disability Studies als interdisziplinäres Forschungsfeld. In: Degener, T./ Diehl, E. (Hg.): Handbuch Behindertenrechtskonvention. Teilhabe als Menschenrecht - Inklusion als gesellschaftliche Aufgabe. Bonn: Bundeszentrale für politische Bildung, S. 334-344.

Walsh, S. (2014): Critiquing the politics of participatory video and the dangerous romance of liberalism. In: Area 48 (4), S. 405-411.

\section{Internetseiten}

Heino Deckert (Q\&A - The Queen of Silence) | One World Romania 2015, https://www.youtube.

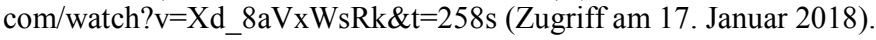

Festival dei Popoli 2015 - Interview with Agnieszka Zwiefka, https://www.youtube.com/watch? $\mathrm{v}=\mathrm{PFcD} 96 \mathrm{BgqE} 8 \mathrm{t}=405 \mathrm{~s}$ (Zugriff am 17. Januar 2018).

Katie chats: hotDOCS, Agnieszka Zwiefka, filmmaker, The Queen of Silence 13.05.2015, https:// www.youtube.com/watch?v=wY7yfk6u0MI\&t=4s (Zugriff am 17. Januar 2018).

\section{Filmografie}

Carayol, S./Callan, K. (2016): Sound System. Frankreich: arte, https://www.arte.tv/de/videos/RC014285/sound-system/ (Zugriff am 17. Januar 2018).

Scheffner, P./Colorado V. (2016): And-Ek Ghes... . Deutschland: pong Film, RBB. Online abrufbar auf der Homepage der Bundeszentrale für politische Bildung, http://www.bpb.de/media thek/239928/and-ek-ghes-eines-tages- (Zugriff am 17. Januar 2018).

Zwiefka, A. (2014): The Queen of Silence. Polen, Deutschland: Ma.Ja.De Filmproduktion, Chilli Productions. In Ko-Produktion mit HBO Europe, Stefilm International, Odra-Film und ZDF. DVD: Deckert Distribution. 